(3) Keeling JW: Congenital and developmental abnormalities. The small intestine. In Whitehead R (ed): Gastrointestinal and Oesophageal Pathology. 2nd ed. Edinburoh. Churchill Livingstone. 1995; pp 289-301.

(4) Morson BC. Dawson IMP. Day DW, Jass JR. Price AB. Williams GT: Meckel's diverticulum, in Morson BC. Dawson IMP. Day DW, Jass JR, Price AB, Williams GT (eds): Morson \& Dawson's Gastrointestinal Patholory. 3rd ed. Oxford, Blackwell Scientific Publications, 1990: pp 222-223.

(5) Moyana TN: Carcinoid tumors arising from Meckel's diverticulum. A clinical, morphological, and immunohistochemical study. Am J Clin Pathol 1989:91:52-56. (6) Schmid KW, Brink M. Freytag G. Kirchmair R, Böcker W. Fischer-Colbrie R. Heitz PU, Klöppel G: Expression of chromogranin $A$ and $B$ and secretoneurin in neoplastic and nonneoplastic pancreatic alpha cells. Virchows Archiv 1994;425:127neoplast 132 .

(7) Soltero MJ, Bill AH: The natural history of Meckel's diverticulum and its relation to incidenta! removal. A study of 202 cases of diseased Mecke!'s diverticulum found in Kings County. Washington, over a fifteen-year period. Am I Surg 1976;132:168174

(8) Sungler P, Hacker GW. Dietze O, Boeckl O: Eine inkarzerierte, maligne Littrésche Hernie: Rarität in der Leistenbruchchirurgie. Acta Chirurgica Austriaca 1997:29:355-359.

(9) Weinstein EC. Dockerty MB, Waugh LJM: Neoplasms of Meckel's diverticulum. Int Abstr Surg 1963;116:103-111

\section{Schlußwort der Autoren}

Das angewandte operative Vorgehen ist daraus zu erklären, daß es sich im vorliegenden Fall um einen Zufallsbefund ohne vorhergegangene, intraoperative histologische Untersuchung handelte. Erst in der nachfolgenden, exakten histopathologischen Aufarbeitung hatte sich gezeigt, daß es sich um einen neuroendokrinen Tumor handelte.

In dieser Publikation wurde absichtich einiges nicht erwähnt, um den Artikel kurz und prägnant zu halten. So haben wir naturlich genaue immunhistochemische Untersuchungen auf eine Reihe von Markern durchgeführt, die $K$.W. Schmid (4) erwähnt. Dazu gehörten unter anderem die generellen Gewebemarker Chromogranin A, Synaptophysin, PGP-9.5, S-100-Proteine, Cytokeratine und eine Reihe möglicher spezifischer Produkte wie

Korrespondenzanschrift: Prof. Dr. G. Hacker, Labor für Immunhistochemie und Biochemie, Institut für Pathologische Anatomie, Landeskrankenanstalten Salzburg, Müllner Hauptstraße 48, A5020 Salzburg.
Serotonin und regulatorische Peptide. Zusätzlich wurden Silberverfahren wie Masson-Argentaffinität, Grimelius- und Servier Munger-Argyrophilie verwendet, um den Tumor genauestmöglich abzuklären (2). All dies erfolgte retrospektiv, denn wie be reits erwähnt, war aus verschiedenen Gründen keine intraoperative Gefrierschnittdiagnostik durchgeführt worden.

Die Möglichkeit, daß es sich um einen von Magen- oder Pankreasgewebe ausgegangenen Tumor handeln könnte, wurde durch zusätzliche Immunhistochemie weitergehend untersucht. Neben Serotonin und Substanz P fanden sich keinerlei weitere Reaktionen fuir spezifische Produkte wie Amine oder Peptide. So waren auch Gastrin, Insulin, Glucagon, Somatostatin, humanes pankreatisches Polypeptid (hPP), Amylin, vasoaktives intestinales Polypeptid (VIP), Helospectin und Pituitary Adenylate Cyclase Activating Peptide (PACAP) nicht nachweisbar, wodurch sich mit relativer Sicherheit ein Ursprung aus dem Pankreas ausschließen läßt

Das mögliche Vorliegen eines ECL-Zell-Tumors (ECLoma) aus dem Magen, der in der Literatur als serotoninnegativer neuroendokriner Tumor diskutiert wird und keineswegs mit dem Masson/Serotonin-positiven EC-Zell-Tumor des Gastrointestinaltraktes verwechselt werden sollte, wurde durch positive Färbungen von Serotonin, Masson-Argentaffinität und GrimeliusArgyrophilie eindeutig ausgeschlossen $(1,2,3)$. ECLome zeigen im übrigen sehr oft einen recht günstigen klinischen Verlauf.

\section{Literatur}

(1) Solcia E, Bordi C. Creutzfeldt W, Dayal Y, Dayan AD. Falkmer S, Grimellis L. Havu N: Histopathological classification of nonantral gastric endorine growths in man. Digestion 1988:41:185-200.

(2) Grimelius L, Su H. Hacker GW: The use of silver stains in the identification of neuroendocrine cell types. In Gu J, Hacker GW (eds): Modern Methods in Analytical Morphology. New York, Plenum Press 1994, pp 1-8.

(3) Hacker GW, Muss WH. Graf AH, Graf B. Hauser-Kronberger C. Dietze O, Sonnteitner-Wittauer U, Su H, Sundler F, Grimelius L: Helodermin/helospectin-like immunoreactivity in an enterochromaffin-like cell tumor (ECLoma) of the stomach. Cell Vision 1996:6:424-428.

(4) Schmid KW: Eingeladener Kommentar zu: Eine inkarzerierte, maligne Littrésche Hernie: Rarität in der Leistenbruchchirurgie. Acta Chirurgica Austriaca 1997:29: 355-359

\title{
Kongreßankündigung
}

\section{Austrian Society of Hematology and Oncology}

\section{INTERNATIONAL SYMPOSIUM}

\section{HEMATOLOGY 1998 - STATE OF THE ART}

Termin und Ort: 21. Februar 1998 - Grand Hotel Wiesler, Grieskai 4, A-8020 Graz

\section{Welcome:}

Chair: 8.30 Uh 9.00 Uhr 9.15 Uhr $9.45 \mathrm{Uhr}$ 10.00 Jhr

Chais:

$10.45 \mathrm{Uhr}$ $11.15 \mathrm{Uhr}$ 11.30 Uhr $12.00 \mathrm{Uhr}$

\section{W. Linkesch (Graz)}

W. Linkesch (Graz), H. Gadner (Wien) A. Bumett (Cardiff): Therapy of AMI Discussant: $K$. Lechner (Wien) D. Höleer (Frankfurt): Therapy of ALL Discussant: $M$. Wernil (Aarau) Consensus Pane?

A. Bumett, H. Gadner, D. Hölzer,

K. Lechner, W. Linkesch. M. Wernli

W. Hinterberger (Wien), D. Niederwieser (Innsbruck)

J. Goldman (London): Therapy of CML Discussant: F. Guilhot (Poitiers)

A. Ganser (Hannover): Therapy of MDS Discussant: E. Hellström Lindberg (Huddinge)

\subsection{Uhr Consensus Panel}

A. Ganser, J. Goldman, F. Guilhot,

E. Hellström Lindberg. W. Hinterberger.

D. Niederwieser

Chair:

13.30 Uhr

$14.00 \mathrm{Uhr}$

14.15 Uhr

$14.45 \mathrm{Uhr}$

$15.00 \mathrm{Uhr}$

D. Lutz (Linz), H. Huber (Wien)

J. L. Harousseau (Nantes): Therapy of MM

Discussant: $H$. Ludwig (Wien)

J. L. Binet (Paris): Therapy of CLL

Discussant: G. Gastl (Innsbruck)

Consensus Panel

J. L. Binet, G. Gastl, J.L. Harousseau,

H. Huber, H. Ludwig, D. Lutz

Chaำ:

15.30 Uhr A. Goldstone (London): Therapy of hm (Wien) NiL

36.00 Uhr M. Kaminski (Ann Arbour): Therapy with Monocional Antibodies in NiL

16.30 Uhr $\quad H$. Tesch (Köln): Therapy of M. Hodgkin

17.00 Uhr Discussant: N. Schmitz (Kiel)

17.15 Uhr Consensus Panel

D. Geissler, A. Goldstone, U. Jäger,

M. Kaminski, E. Pittermann, N. Schmitz.

H. Tesch

This symposim is supported by an educational grant irom AMGTN Gmb Vienna. 